\title{
Influence of tongue movements on masticatory efficiency
}

\author{
Hiroko Oguchi, Taihei Watanabe, Norimitsu Nakamura and Shigeru Watanabe* \\ Division of Pediatric Dentistry, Department of Human Development and Fostering, Meikai University School of Dentistry, 1-1 Keyakidai, Sakado, Saitama, Japan
}

\begin{abstract}
Introduction: The purpose of this study is to test relationships between tongue movements and electromyographic activity (EMA) of suprahyoid muscles.

Methods: The subjects were 6 adults with a normal stomatognathic system. Experiment 1: Each subject performed six types of tongue movement: 1 . extrusive, 2 . right-and-left lateral, 3. upward, 4. downward, 5. touching the palate with the tongue tip, 6. touching the palatal aspects of the maxillary second molars, while EMAs were recorded with a wireless electrode-type system in the suprahyoid, digastric and masseter muscles. Experiment 2: Tongue movement was restricted with a device fitted into the floor of the mouth. Then compared were masticatory efficiency and EMA when tongue movements were (a) restricted, (b) not restricted, and (c) when free mastication was allowed without use of the tongue movement restriction. Masticatory efficiency was measured as by Manly RS, et al., [1].

Results: In Experiment 1, EMAs of all tongue movements were significantly higher than resting EMAs. In Experiment 2, masticatory efficiencies and EMAs of (a) were significantly lower than those of (c). Comparing (a) and (c), 3 test subjects in (a) had significantly longer masticatory cycles. Comparing (b) and (c), no significant difference was found in either masticatory efficiency or EMA. In (c), a significant positive correlation was found between masticatory efficiency and the EMA of the suprahyoid muscles.
\end{abstract}

Conclusion: EMA of the suprahyoid muscles reflected the tongue movements and the tongue movements significantly influence masticatory efficiency.

\section{Introduction and proposition}

The timing of swallowing of a food bolus has been termed 'the swallowing threshold' by Shiere, et al., [2] Although there are interindividual differences in swallowing thresholds, a study has shown that there are hardly any intraindividual differences in chewing rhythm and swallowing threshold [3]. In addition to food comminution and number of masticatory strokes [2-4], water content to adjust food consistency $[5,6]$ is also involved in food bolus formation during mastication. This suggests that teeth and saliva are important in determining swallowing thresholds.

On the other hand, few studies have provided evidence that the tongue significantly influences masticatory efficiency [7]. Nakasima [8] reported that the comminution index decreased when subjects were instructed to chew while wearing a bed apparatus fitted with a protrusion to restrict tongue movement toward the lingual side of the bilateral mandibular premolar regions. Furthermore, in a study that ultrasonographically evaluated tongue movement capability during mastication [9], tongue movement dynamics were difficult to analyse quantitatively, and the details of the relationship between tongue movement during mastication and masticatory efficiency were not clarified. As for the relationship between tongue movement and the suprahyoid muscles, Sasaki, et al., [10] evaluated five basic movements of the tongue left, right, forward, opening and saliva swallowing movements using a nine-channel electrode array and reported that the action potential of the suprahyoid muscles reflected the tongue movements with a high probability.

Therefore, we aimed to clarify the effects of tongue movement on mastication by measuring electromyographic activities (EMAs) of suprahyoid muscles during mastication and examining their relationship with masticatory efficiency.

\section{Methods}

The present study was approved by the ethics committee of Meikai University School of Dentistry (approval number: A 1216). This study was conducted in full accordance with the World Medical Association Declaration of Helsinki.

\section{Paticipants and procedure}

Experiment 1: Relationship between EMAs of suprahyoid muscles and tongue movement

The subjects were six orally healthy adults with a normal stomatognathic system (three men, three women; age 26-35 years; mean age $30.3 \pm 2.8$ years). We obtained written informed consent from all the subjects before the initiation of the study.

Surface electrodes $(30 \times 24 \mathrm{~mm}$ in diameter; KendallTM H124SG, COVIDIEN, Neustadt an der Donau, Germany) were applied symmetrically to each subject's skin on the right and left suprahyoid muscles (including the digastric muscles) at $20-\mathrm{mm}$ intervals (Figures la and $1 \mathrm{~b}$ ). The range of frequencies between 16 and $500 \mathrm{~Hz}$ was recorded.

The EMA measurements were loaded into a personal computer

Correspondence to: Shigeru Watanabe, Department of Human Development and Fostering, Meikai University School of Dentistry, 1-1 Keyakidai, Sakado, Saitama 350-0283, Japan, Tel: (+81) 49-279-2743, Fax: (+81) 49-279-2743, E-mail: shigeru@dent.meikai.ac.jp

Key words: surface electromyography, suprahyoid muscles, tongue movements, masticatory efficiency, peanuts, masticatory cycles

Received: November 22, 2016; Accepted: December 07, 2016; Published: December 09, 2016 
using four channels (FREEEMG-RT; BTS, Milan, Italy) from a wireless surface electrode-type system after $\mathrm{A} / \mathrm{D}$ conversion at a $1-\mathrm{kHz}$ sampling rate. The loaded values were analysed using EMG analysis software (EMG Analyzer, Version 1.7.17.0; BTS). We measured EMAs of six types of tongue movement performed by the subjects: 1. rightand-left lateral movement; 2 . touching the palate with the tongue tip; 3 . touching the palatal aspects of the maxillary second molars; 4. extrusive movement; 5. upward movement (subnasal point) and 6. downward movement (skin on the mental region). The results of 1,2, and 3 (Figure 2) were compared with resting EMAs with the mouth closed, and the results of 4, 5, and 6 (Figure 3) were compared with resting EMAs with the mouth open (for each tongue movement). Each subject performed the six tongue movements three times. To analyse the mean values of the subjects, we used the 10-s EMG readings (the intermediate value between 5 and $15 \mathrm{~s}$ ), which were considered the most stable values.

Experiment 2. Relationship between EMAs of suprahyoid muscles and masticatory efficiency.

The subjects were six healthy adults (one man, five women; age 2535 years; mean age $30.3 \pm 3.1$ years) selected by the same conditions as in Experiment 1.

Tongue movement was restricted with a device fitted into the floor of the mouth. Using a dental alginate impression material and tray for the upper jaw, we took impressions of the lower jaw including the tongue fitted into the floor of the mouth. Then, we made a plaster

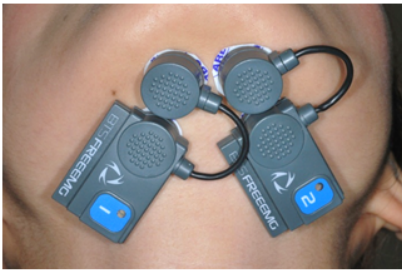

(a)

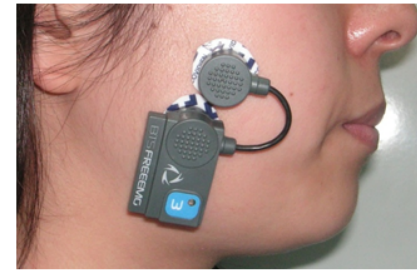

(b)

Figure 1. Placement of the electrodes. a) Anterior belly of the suprahyoid muscles. b) Middle part of the masseter muscle.

(1)
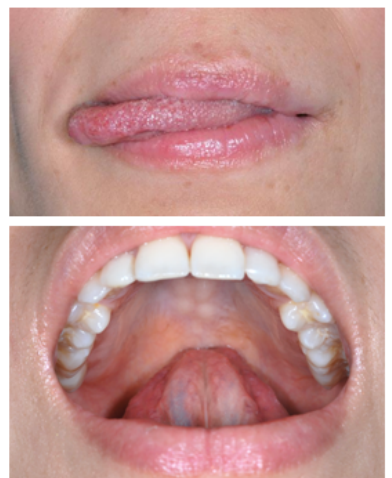

(2)
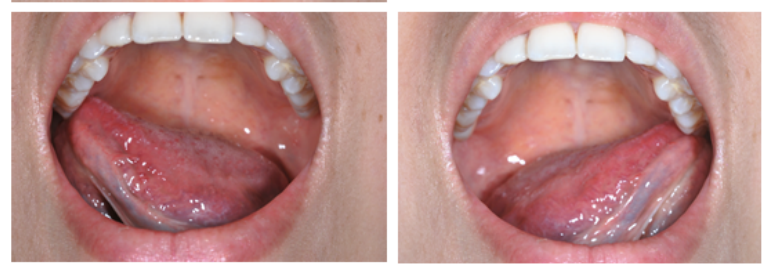

Figure 2. Tongue movements. (1) Right-and-left lateral extrusion. (2) Touching the palate with the tongue tip. (3) Touching the palatal aspects of the maxillary second molars.
(4)

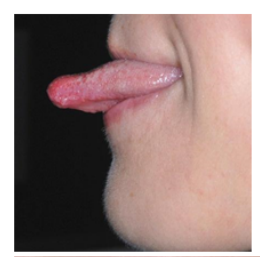

(5)

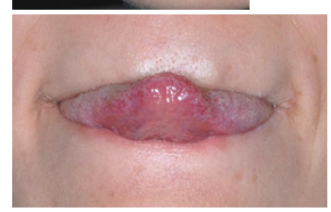

(6)

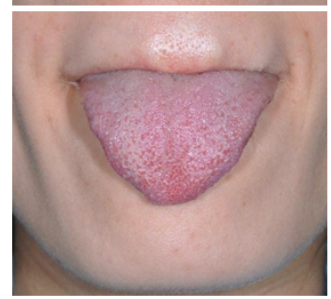

Figure 3. Tongue movements. (4) Protrusive extrusion. (5) Upward extrusion (tongue tip on subnasal point). (6) Downward extrusion (tongue tip on mental region).

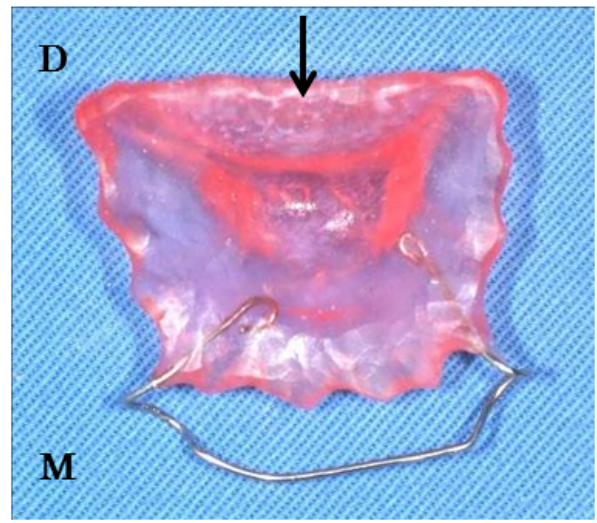

Figure 4. Device on the floor of oral cavity (M: Mesial, D: Distal). A tip of the tongue enters under the arrow of the device.

model for each subject and covered the tongue (the region from anterior teeth to first molar) with a $1.5-\mathrm{mm}$-thick resin (dental quick cure resin; Nissin, Tokyo, Japan). We then placed and maintained a labial wire $(0.7 \mathrm{~mm}$ in diameter) between the right and left canine teeth of the lower jaw (Figure 4).

Each subject performed masticatory movements five times under the following three conditions: Tongue movement was restricted with the device (the tongue tip was inserted under the resin plate) (Figure $5 \mathrm{a}$ ). Tongue movement was not restricted with the device (the tongue was placed on the resin plate) (Figure 5b). Tongue movement was not restricted; this was done without the device.

We measured the EMAs from the right and left suprahyoid muscles, including the digastric and masseter muscles (Figure 1b), in the same manner as in Experiment 1 . In order to evaluate masticatory cycles, we determined the beginning and end of mastication. The EMG analyser and measurement conditions were the same as in Experiment 1. The test food was $3 \mathrm{~g}$ of peanuts (eight half peanuts with no plumule and radicle; unsalted roasted peanuts; Fukuya, Yachimata, Chiba, Japan).

Each subject chewed the test food freely 20 times (free mastication); during chewing, we calculated EMAs and masticatory efficiencies. The 


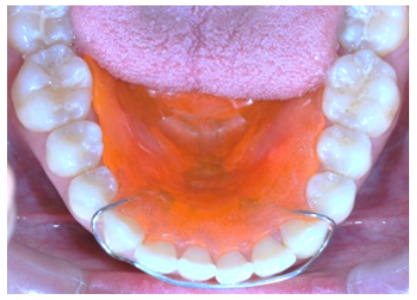

(a)

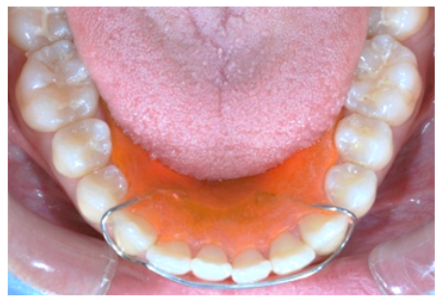

(b)
Figure 5. Tongue restricting device fitted into the floor of the mouth. a) Limited tongue movements (A tip of the tongue is beneath the device). b) Unlimited tongue movements (A tongue is on the device).

EMAs were measured in the same way as in Experiment 1, whereas the masticatory efficiencies were evaluated using a method by Manly, et al., [1]. We prepared $15 \mathrm{~g}$ of peanuts (test food) and divided the subjects into five groups. Each group chewed the test food $(3 \mathrm{~g}) 20$ times. In addition, we used an electric drying oven (FS-620, Advantec Toyo, Tokyo, Japan) to dry particles retained on a 10-mesh sieve (sieve for analysis, Ikemoto Scientific Technology, Tokyo, Japan) at $100^{\circ} \mathrm{C}$ for 3 $\mathrm{h}$. The dried particles were measured using a digital analytical balance (HR-60, Kensei Kogyo, Shimotsuma, Ibaraki, Japan) to calculate masticatory efficiency.

Free mastication in this experiment implied masticatory patterns naturally performed by the subjects with no instruction on the use of the right or left anterior molar region [11]. We required the subjects to close their mouths and not to swallow the peanuts during mastication.

After all the subjects performed the above experiment five times, we analyzed the subjects' mean EMA values. The beginning/end of an EMA from the masseter muscle was regarded as the beginning/end of mastication. The EMAs were integrated between 20-time masticatory strokes after all EMG waveforms were corrected for the absolute values.

The masticatory efficiency was calculated by the following method:

masticatory value $=[$ (total dry weight $[\mathrm{g}]-$ sieve dry residue $[\mathrm{g}]$ ) / total dry weight $[\mathrm{g}]] \times 100$

However, we assumed that total dry weight was 98 [\%] of wet weight.

masticatory efficiency $=(20$ [times] $/$ number of masticatory strokes required for 78 [\%] of the sample to pass through the 10-mesh sieve) $\times 100$

We measured the duration and intervals of EMG discharge from EMA potentials of the masseter muscles during mastication and compared masticatory cycles [12] (Figure 6) under conditions of tongue restriction and free mastication.

\section{Statistical analyses}

First, we conducted Wilcoxon tests on the EMAs of the suprahyoid and masseter muscles in Experiment 2 and compared the masticatory cycles between the restricted-tongue and free-mastication groups. Second, we performed one-way analysis of variance and Dunnett's tests (multiple comparison procedures) on the EMAs of the tongue movements in Experiment 1 and compared the results with resting EMAs with the mouth closed and open. Finally, we implemented oneway analysis of variance and Tukey's honestly significant difference tests on the masticatory efficiency and EMAs in Experiment 2 and compared the differences among the restricted-tongue, non-restricted- tongue, and free-mastication groups. In Experiment 2, the masticatory efficiency and EMAs under free mastication conditions were calculated using the Pearson correlation coefficient.

The statistical software used was KaleidaGraph (Version 4.1.0, Windows 7; Hulinks, Tokyo, Japan).

In order to obtain the integral values (Integrated EMGs; IEMGs) of the suprahyoid muscles during the 10-s tongue movements as EMAs, the EMGs recorded in Experiment 1 were analysed using the analysis software of an EMG recording device.

The IEMGs of the suprahyoid muscles, including the masseter, during 20-time mastication were calculated as EMAs in Experiment 2, using the same software as in Experiment 1. When determining the masticatory cycles in the 20-time mastication, we used the results obtained at five strokes (the intermediate value of 8-12 strokes) which were considered stable mastication values. The mean values and standard deviations were then calculated.

\section{Results}

Experiment 1: Relationship between EMAs of suprahyoid muscles and tongue movement.

Figure 7 shows the mean values of all the subjects' EMAs of the suprahyoid muscles with their mouths closed and then during the tongue movements 1,2 , and 3 . The EMAs during the tongue movements significantly increased ( 2 was $\mathrm{p}<0.05 ; 1$ and 3 were $\mathrm{p}<0.001$ ), compared

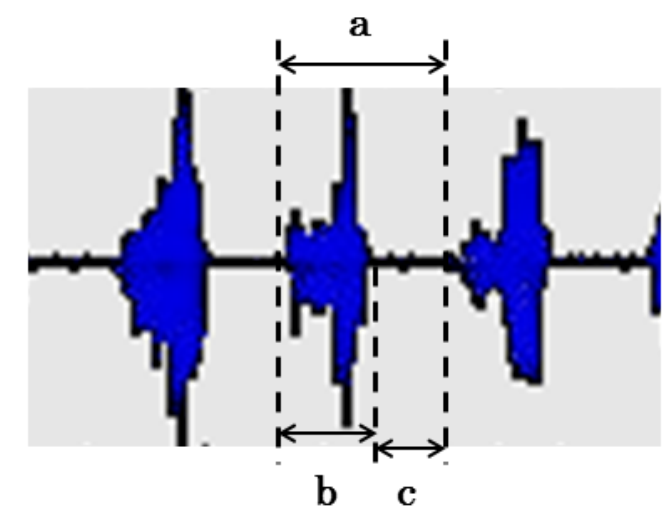

Figure 6. Chewing cycle at the time of the masseter muscle activity (a: Cycle time, b: Duration, c: Interval).

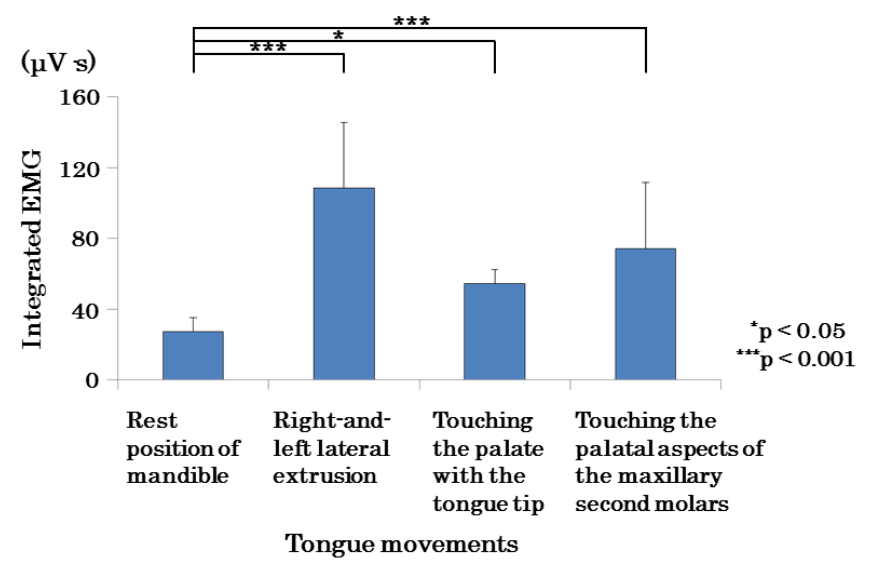

Figure 7. Comparison of mean values of EMA in the suprahyoid muscles in rest position of mandible and tongue movements. 
to the resting EMAs with the mouth closed.

Figure 8 shows the mean values of all the subjects' EMAs of the suprahyoid muscles with their mouths open and during the tongue movements 4,5 , and 6 . The EMAs during all of the tongue movements $(4,5$, and 6) significantly increased $(\mathrm{p}<0.001)$ compared with the resting EMAs with open mouths.

Experiment 2: Masticatory efficiency and EMAs.

Figure 9 shows the mean values of the subjects' masticatory efficiencies analysed in the three types of experiments: significant differences $(p<0.001)$ were observed in masticatory efficiencies between the restricted-tongue and free-mastication groups.

Figure 10 shows the suprahyoid muscle EMAs. Similarly, significant differences (sub 3, 4 , and 5 were $\mathrm{p}<0.01$; sub 1,2 , and 6 were $\mathrm{p}<0.001$ ) were observed between the restricted-tongue and free-mastication groups and no significant differences between the non-restrictedtongue and free-mastication groups.

Table 1 lists the mean values of the subjects' masticatory efficiencies and suprahyoid muscle EMAs. Significant differences $(\mathrm{p}<0.001)$ were found between the restricted-tongue and free-mastication groups, but not between the non-restricted-tongue and free-mastication groups.

The suprahyoid muscle EMAs were significantly different $(\mathrm{p}<0.001)$ between the restricted-tongue and free-mastication groups, but not between the non-restricted-tongue and free-mastication groups.

The mean values of the subjects' masticatory cycles (Figure 11)

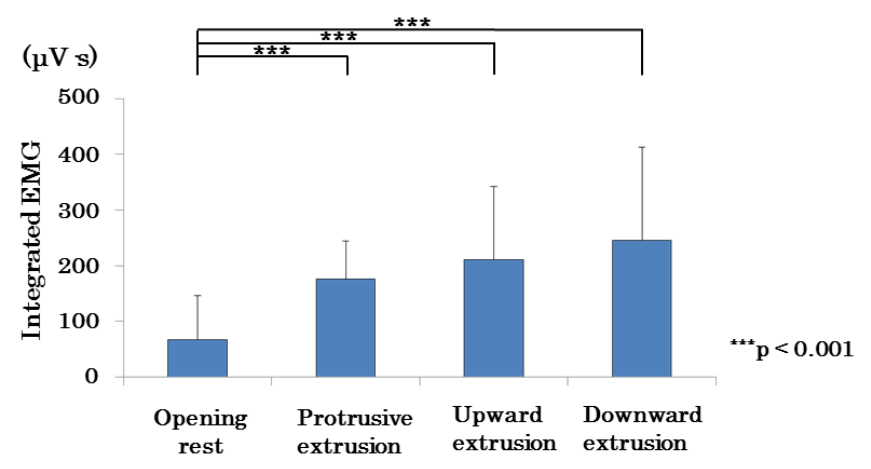

Tongue movements

Figure 8. Comparison of mean values of EMA in the suprahyoid muscles in opening rest and tongue movements.

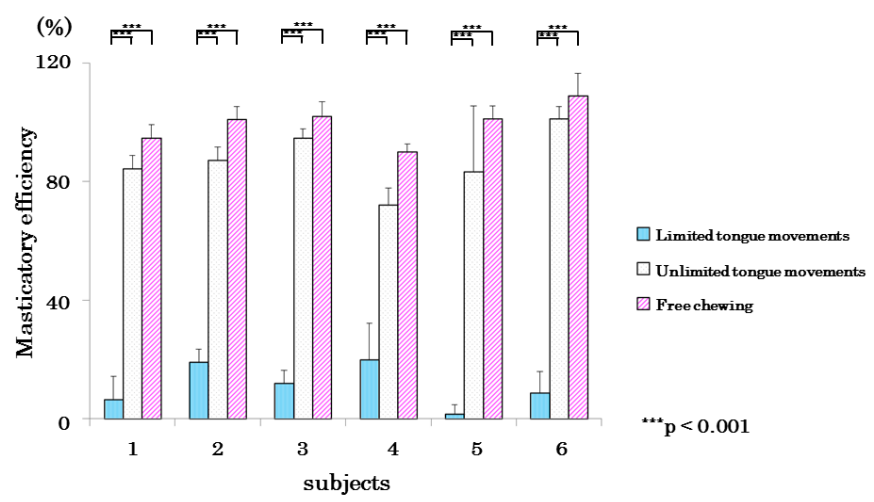

Figure 9. Comparison of mean values of in the masticatory efficiency in limited, limited tongue movements, and free chewing in each subject.

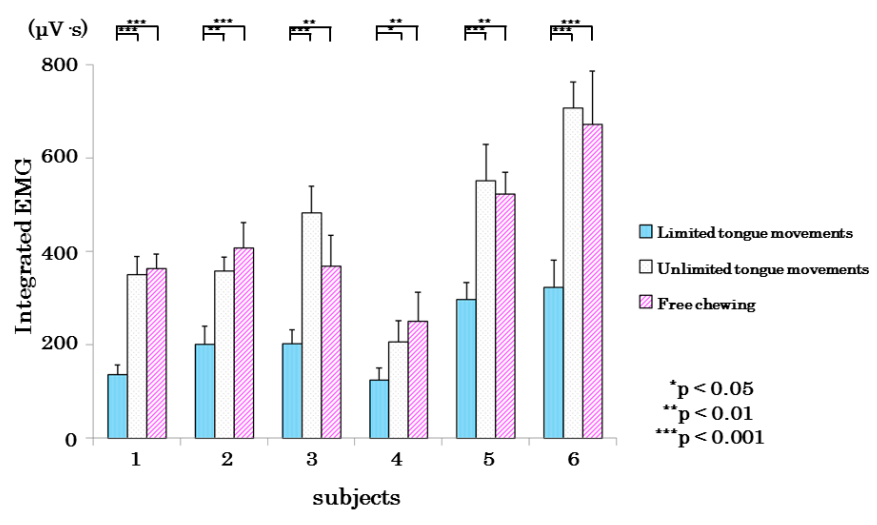

Figure 10. Comparison of mean values of EMG activity in the suprahyoid muscles in limited, unlimited tongue movements, and free chewing in each subject.

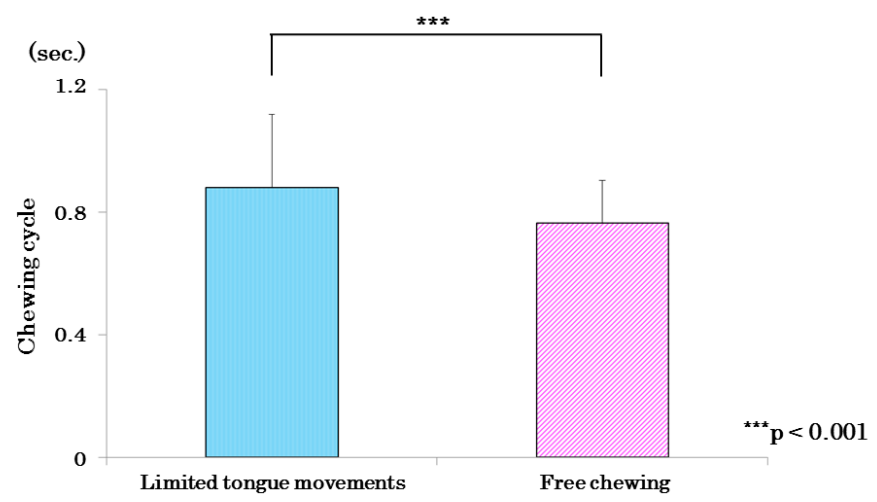

Figure 11. Comparison of mean values of chewing cycle in limited of tongue movements and free chewing.

were significantly longer in the restricted-tongue group than in the free-mastication group.

Figure 12 indicates the percentages of the masticatory cycles of the restricted-tongue group when the masticatory cycles of the free-mastication group were $100 \%$. As a result of comparison of the masticatory cycles between the restricted-tongue and free-mastication groups, the subjects $4,5(\mathrm{p}<0.001)$, and $6(\mathrm{p}<0.05)$ showed significantly longer cycles when tongue movements were restricted.

In this study, the EMAs of the suprahyoid muscles were significantly and positively correlated $(\mathrm{p}<0.05)$ with the masticatory efficiency under free-mastication conditions (Figure 13).

\section{Discussion}

\section{Relationship between tongue movement and the suprahyoid muscles}

In Experiment 1 of the present study, we examined the relationship between tongue movement and suprahyoid muscle activity as a means of evaluating tongue movement. Comparisons of tongue movement and resting muscle activity with the mouth closed and tongue movement with and muscle activity with the mouth open beforehand in a position in which the movement could be performed revealed significant differences between the mean values of both. This indicates that measurement of suprahyoid muscle activity is useful as a method to indirectly evaluate tongue movement.

The tongue movement with the mouth closed, as performed in 
Table 1. Comparison of mean values of masticatory efficiency and integrated EMG.

\begin{tabular}{|c|c|c|c|c|c|c|}
\hline & \multicolumn{3}{|c|}{ Masticatory efficiency (\%) } & \multicolumn{3}{|c|}{ Integrated EMG $(\mu \mathrm{V} \cdot \mathrm{s})$} \\
\hline & $\begin{array}{l}\text { Limited tongue } \\
\text { movements }\end{array}$ & $\begin{array}{l}\text { Unlimited tongue } \\
\text { movements }\end{array}$ & Free chewing & $\begin{array}{l}\text { Limited tongue } \\
\text { movements }\end{array}$ & $\begin{array}{l}\text { Unlimited tongue } \\
\text { movements }\end{array}$ & Free chewing \\
\hline $\begin{array}{l}\text { Mean } \\
\text { (S.D.) }\end{array}$ & $\begin{array}{l}11.21^{* 1} \\
(10.90)\end{array}$ & $\begin{array}{l}87.15 \\
(10.07)\end{array}$ & $\begin{array}{l}99.74^{22} \\
(9.94)\end{array}$ & $\begin{array}{l}214.05 \\
(84.72)\end{array}$ & $\begin{array}{c}443.00 \\
(170.00)\end{array}$ & $\begin{array}{l}431.04{ }^{* 2} \\
(150.43)\end{array}$ \\
\hline
\end{tabular}

Significant differences were found between ${ }^{*_{1}}$ and ${ }^{*_{2}},{ }^{* * 1}$ and ${ }^{* *_{2}}(\mathrm{p}<0.001)$.

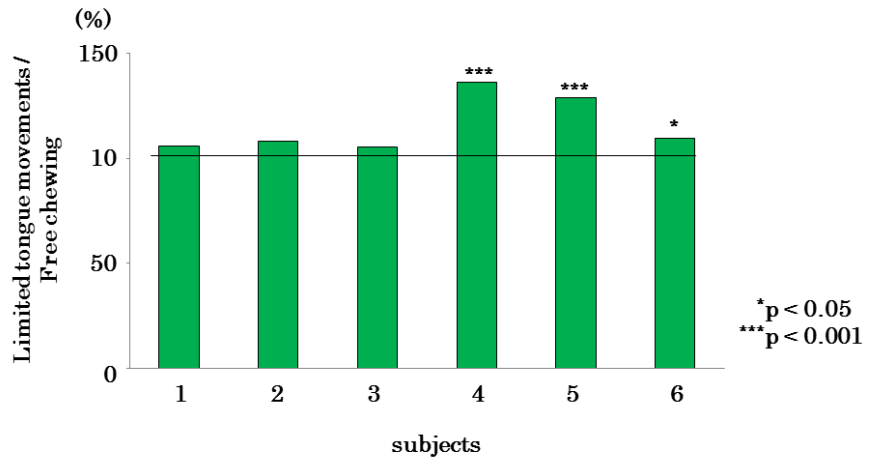

Figure 12. Comparison of mean values of EMG activity in the suprahyoid muscles in limited, unlimited tongue movements, and free chewing in each subject.

the present experiment, appears to be closer to movement during mastication than movement with the mouth open. As Nakasima [8] stated that the tongue selects relatively large particles within food boluses to send to the occlusal surface during mastication, tongue movement during mastication is also responsible for assisting comminution function by carrying food to the occlusal surface. Tongue movement measured in the present study that exhibited the greatest difference compared to action potential with the mouth closed in the resting position involved muscle activity during lateral movement of the tongue. This result indicates that the suprahyoid muscles activity reacts sensitively to these movements.

\section{Measurement methods of masticatory efficiency}

According to Hashimoto [11], in the peanut mastication method, comminution of the test food is affected by water content, while in the sieve method that uses water, the measured value is reported to become more accurate with lower water content of the water-soluble components within the food. We conducted a preliminary experiment in the aim to examine the dry weight of peanuts [13] to address this issue in the peanut mastication method. We found that the total dry weight was $98 \%$ of the original weight $(3.0 \mathrm{~g})$, suggesting that changes in the shape of peanuts due to water absorption that affected passage through a sieve is within a negligible range.

Comparisons under the same conditions and within the same individual subjects were performed in the present study, which thereby led us to believe that the level of masticatory efficiency can be determined on the basis of fluctuations in tongue movement.

\section{Masticatory efficiency and masticatory cycles}

In Experiment 2 of the present study, the suprahyoid muscles activity during mastication and masticatory efficiency were measured on the basis of the results of Experiment 1 and the preliminary experiment to indirectly evaluate tongue movement during mastication. For the measurements, mastication experiments were conducted while subjects wore an apparatus to restrict tongue movement in order to understand the muscle activity involved in movement during opening

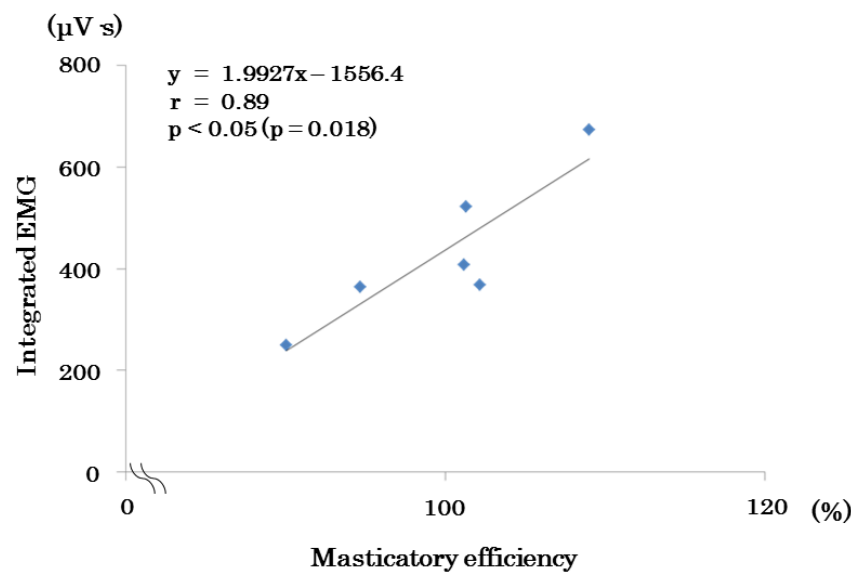

Figure 13. Scatter plots of masticatory efficiency and EMA in the suprahyoid muscles during free chewing.

and closing of the jaw. The results of these experiments revealed that, during restriction of tongue movement, suprahyoid muscles activity decreased to an average of approximately $50 \%$ compared with normal free mastication, while masseter muscle activity decreased to an average of approximately $59 \%$. This indicates that when subjects are instructed to chew while tongue movement is restricted by fixating a tongue within the apparatus, suprahyoid muscles activity involved in mouth opening was at approximately $50 \%$. Needless to say, masticatory efficiency also decreased to approximately $11 \%$ under these conditions, which is a marked reduction compared to that with free mastication.

Thus, although mastication performed with almost all movements of the tongue restricted by an apparatus is an experiment outside the scope of day-to-day expectations, we measured masticatory cycles in order to understand how mastication without tongue involvement works.

Masticatory cycles primarily evaluate masticatory rhythms and can be obtained by analysing the time taken between the start of muscle discharge and the next using an electromyogram [12]. Sugawara [14] reported that masticatory cycles were prolonged during experimentally created nasal congestion conditions. As demonstrated in the present experiment, significant prolongation of masticatory cycles was seen in three of six subjects during mastication where tongue movement was restricted. The masticatory cycles of the other three subjects also tended to be longer and significant prolongation of masticatory cycles was seen in overall mean values [15-17].

This indicates that masticatory rhythm is more irregular during tongue restriction than during free mastication.This disturbed masticatory rhythm was probably due to food boluses not being carried efficiently to the occlusal surface by the tongue, leading to a lower comminution index.

\section{Conclusion}

We investigated the relationship between tongue movement and 
suprahyoid muscles activity and the effects of tongue movement on masticatory efficiency. Our results demonstrated that suprahyoid muscles activity measurement serves as a useful means of indirectly evaluating tongue movement, and indicated that masticatory cycles are prolonged and masticatory efficiency is markedly reduced when mastication and tongue movement are restricted.

\section{Acknowledgements and conflicts of interest}

The authors would like to thank Prof Kazuyo Muramoto, Prof. Osamu Amano and Prof. Masaki Fujisawa for their guidances to conduct this work, and members in the Division of Pediatric Dentistry, Department of Human Development and Fostering for their supports to the experiment. This work was supported by the Miyata Research Fund 2014, in Meikai University School of Dentistry. 1-1 Keyakidai, Sakado, Saitama 350-0283, Japan. There are no conflicts of interest.

\section{References}

1. Manly RS, Louise CB (1950) Masticatory performance and efficiency. J Dent Res 29: 448-462. [Crossref]

2. Shiere FR, Manly RS (1952) The effect of the changing dentition on masticatory function. J Dent Res 31: 526-534. [Crossref]

3. Kawamura Y, Nobuhara M (1957) Studies on masticatory function. The swallowing threshold of persons with normal occlusion and malocclusion. Med J Osaka Univ 8: 241-246.

4. Vinton P, Manly RS (1955) Masticatory efficiency during the period of adjustment to dentures. J Prosthet Dent 5: 477-480.

5. Watanabe S, Dawes C (1988a) The effects of different foods and concentrations of citric acid on the flow rate of whole saliva in man. Archs Oral Biol 33: 1-5. [Crossref]

6. Watanabe S, Ohnishi M, Kawano E (1993) The effects of citric acid and foods on flow rate of whole saliva in five-year-old children. Pediatr Dent J 3: 79-84.

7. Munro RR (1974) Activity of the digastric muscle in swallowing and chewing. J Dent Res 53: 530-537. [Crossref]

8. Nakasima A (1976) An analysis of several physiological factors influencing the masticatory function. J Kyushu Dent Soc 30: 20-36

9. Imai A, Tanaka M, Tatsuta M, Kawazoe T (1955) Ultrasonographic images of tongue movement during mastication. J Osaka Dent Univ 29: 61-69. [Crossref]

10. Sasaki M, Arakawa T, Nakayama A, Yamaguchi M (2012) Method of tongue movement estimation based on suprahyoid muscle coordination. Trans JPn Soc Med Biol Eng 50: 31-37.

11. Hashimoto Y (1959) Comparison of a variety of test foods for masticatory crushability by sieving method. J Stomatol Soc Jpn 26: 648-676.

12. Yamashita S, Sugita D, Matsuo K (2013) Relationship between stage II transport and number of chewing strokes as mastication progresses. Physiol Behav 122: 100-103. [Crossref]

13. Okiyama S, Ikebe K, Nokubi T (2003) Association between masticatory performance and maximal occlusal force in young men. J Oral Rehabil 30: 278-282. [Crossref]

14. Sugawara M (1997) The Study on the influence of nasal obstruction on human masticatory behavior. Jpn j Ped Dent 35: 684-698.

15. Watanabe S, Dawes C (1988b) A comparison of effects of tasting and chewing foods on the flow rate of whole saliva in man. Archs Oral Biol 33: 761-764. [Crossref]

16. Manly RS, Vinton P (1951) Factors influencing denture function. $J$ Prosthet Dent 1 : 578-586. [Crossref]

17. Lucas PW, Luke DA (1983) Methods for analysing the breakdown of food in human mastication. Archives of Oral Biology 28: 813-819. [Crossref]

Copyright: (C2016 Oguchi H. This is an open-access article distributed under the terms of the Creative Commons Attribution License, which permits unrestricted use, distribution, and reproduction in any medium, provided the original author and source are credited. 